\section{Les cellules souches embryonnaires humaines au secours des hépatites fulminantes}

Yves- $\varepsilon$ douard Herpe, Michelle Hadchouel, Anne Weber, Jean-Paul Thiéry, Yacine Laâbi y.દ. Herpe, Y. Laâbi : AbCys SA. M. Hadchouel, A. Weber, J.P. Thiéry : Inserm U804.

Laboratoire de transfert de gènes dans le foie - applications thérapeutiques, Bâtiment Gregory Pincus,

80, rue du Général Leclerc,

94276 Le Kremlin-Bicêtre, France.

yacine.laabi@abcysonline.com

une colonne de charbon végétal activé et une colonne de résine échangeuse d'ions (ayant pour fonction d'adsorber les molécules toxiques), et surtout un bioréacteur hépatique (BRH). Le BRH est en fait une culture en masse d'hépatocytes séparée du plasma du patient par une membrane semi-perméable (Figure 1). Les premiers appareils étaient formés d'une «cartouche » cylindrique contenant quelques centaines de microtubes (d'une longueur de $35 \mathrm{~cm}$ et d'un diamètre de $760 \mu \mathrm{m}$ ) dans lesquels circule le plasma du patient $[2,3]$. La paroi de ces microtubes est une membrane semi-perméable en acéL'hépatite fulminante se traduit par l'effondrement des principales fonctions physiologiques du foie induisant une encéphalopathie avec œdème cérébral mortel en quelques heures. Sans traitement, $80 \%$ des patients décèdent de cette atteinte neurologique irréversible. La transplantation hépatique en urgence reste la meilleure thérapeutique, mais la rareté des greffons hépatiques compatibles immédiatement disponibles sur place explique que la mortalité des patients en attente de greffe de foie se situe entre $10 \%$ et $15 \%$.

Durant la période d'attente entre le diagnostic et la réalisation de la greffe de foie, on dispose de traitements pallia-

\footnotetext{
${ }^{1}$ Les mycotoxines sont des composés chimiques toxiques, produits par certains champignons. II existe de nombreux composés de cette sorte, mais seul un nombre limité se retrouve régulièrement dans les aliments pour l'homme ou les animaux tels que les céréales. II existe cinq groupes de mycotoxines dont les aflatoxines.

${ }^{2}$ Toxine du champignon amanite phalloïde.
}

tifs visant principalement à détoxifier le plasma du patient (hémodiafiltration) et à diminuer l'œdème cérébral et le métabolisme général (hypothermie). Ces traitements sont généralement peu efficaces et ne font que retarder l'échéance fatale. On a donc mis au point des appareils dénommés communément foie bioartificiel (BAL) [1] destinés essentiellement à remédier de manière temporaire à la déficience du foie et à empêcher une dégradation de l'état général du patient pendant cette période d'attente de greffe.

Ces appareils fonctionnent selon le principe de l'hémodialyse: ils comportent

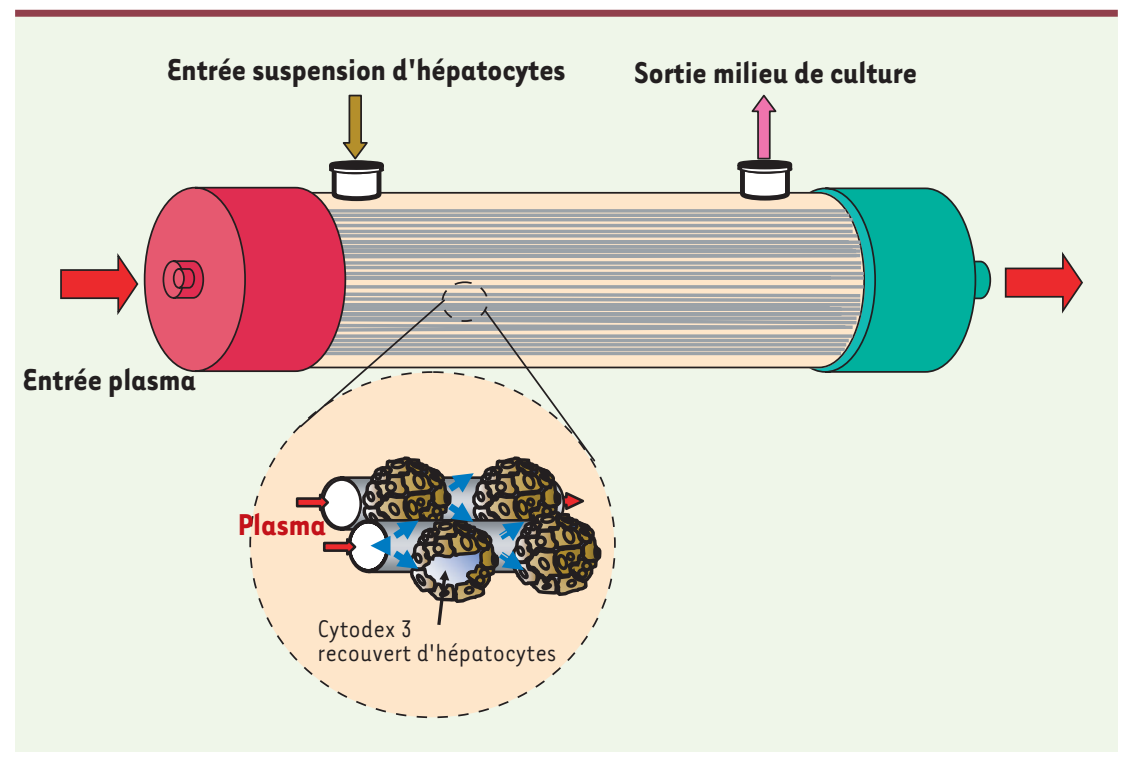

Figure 1. Bioréacteur hépatique de première génération. Les échanges entre le plasma du patient et les hépatocytes s'effectuent à travers la membrane semi-perméable des microtubes (diamètre interne : $760 \mu \mathrm{m}$ ) (système Démétriou [7]). 
tate/nitrate de cellulose permettant les échanges entre le plasma et les cellules hépatiques dans l'espace entre les microtubes. Ces cellules adhèrent à la surface de microbilles de Cytodex 3 , ce qui favorise leurs propriétés fonctionnelles.

Dans les hépatites fulminantes, les fonctions physiologiques du foie natif étant pratiquement inexistantes, le BRH doit contenir au moins $200 \mathrm{~g}$ d'hépatocytes actifs (20\% du foie), soit environ $2.10^{10}$ cellules. Diverses sources d'hépatocytes ont été testées: une lignée continue d'hépatocytes humains en culture (HepG2 clone (3A) d'origine tumorale [2], des hépatocytes humains immortalisés (SV40 ou télomérase) [4], avec le risque de passage de cellules cancéreuses chez le patient, ou des hépatocytes primaires de porc, mais ces cellules hébergent le génome d'un rétrovirus endogène (PERV) qui peut se multiplier dans des cellules humaines, d'où un risque de zoonose [3].

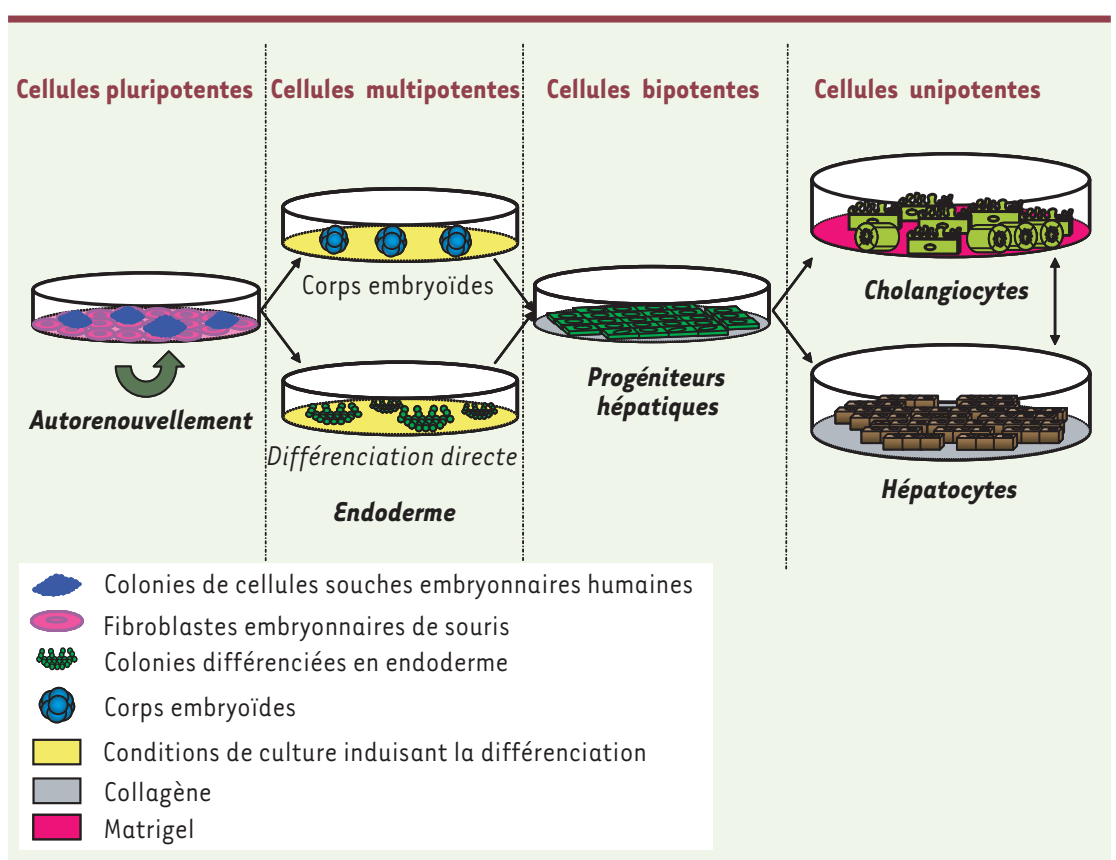

Figure 2. Destinées des hESC selon les conditions de culture. La différenciation des hESC en endoderme peut se faire de manière non contrôlée en passant par les corps embryoïdes. On se retrouve dans ce cas avec un mélange de cellules issues des trois feuillets embryonnaires. On peut également induire la différenciation des hESC de façon massive vers l'endoderme en utilisant des conditions de culture particulières (facteurs de croissance comme l'activine A, agents différenciants, co-culture avec des lignées stromales). À partir de cet endoderme, on isole des progéniteurs hépatiques bipotents qui pourront, selon les conditions de culture, donner des hépatocytes ou des cholangiocytes.
Malgré des résultats très encourageants, les risques encourus par l'utilisation de telles cellules ont conduit les autorités responsables à la suspendre $[6,7]$.

Nous ne disposons pas à l'heure actuelle de lignées d'hépatocytes humains normaux ne comportant pas de risques et pouvant fournir en culture in vitro de telles quantités de cellules. Une possibilité séduisante serait d'utiliser des hépatocytes humains fonctionnels obtenus in vitro à partir de la différenciation de cellules souches embryonnaires humaines (hESC). En effet, sous forme indifférenciée, ces hESC s'autorenouvellent en théorie de manière illimitée in vitro et sont pluripotentes [8]. Les premières lignées d'hESC n'ont été isolées qu'en 1998 [9] et l'on connaît peu de choses sur leur potentiel hépatique. De nombreuses équipes à travers le monde, dont la nôtre, tentent de mettre au point des approches expérimentales permettant méthasone, butyrate de sodium, diméthylsulfoxyde ou trichostatine A) [12]. Les populations cellulaires analysées présentent en effet une morphologie épithéliale et expriment des marqueurs phénotypiques ainsi que des activités enzymatiques typiques d'hépatocytes. Cependant, la fonctionnalité de ces cellules in vivo n'a jamais été démontrée.

Les hépatocytes ayant une origine dans le feuillet embryonnaire endodermique, une autre approche consiste à induire la différenciation séquentielle des hESC d'abord en endoderme définitif, puis en hépatocytes. Chez la souris, la différenciation des cellules souches embryonnaires en endoderme définitif [15] puis en hépatocytes n'a été décrite que très récemment [16]. Cependant, bien que la fonctionnalité de ces cellules ait été suggérée in vitro par des tests biochimiques, leur fonctionnalité in vivo n'a été rapportée que par une seule équipe [17]. Chez l'homme, la nature moléculaire des facteurs inducteurs de l'endoderme demeure largement inconnue. En outre, plusieurs marqueurs de l'endoderme définitif sont aussi exprimés par les tissus extra-embryonnaires, 
en particulier l'endoderme primitif et ses dérivés (endodermes viscéral et pariétal), ce qui complique la caractérisation. Enfin, ces marqueurs sont intracellulaires et ne peuvent donc être utilisés pour isoler les cellules d'intérêt. Un seul article a récemment décrit l'obtention d'endoderme définitif de manière convaincante [18]. En présence d'Activine $A^{3}, 80 \%$ des hESC se différencient en endoderme définitif, et le récepteur CXCR4 (dont le ligand est SDF-1 ou CXCL12) spécifique de ce tissu permet de purifier la population cellulaire d'intérêt. II reste à développer des méthodes efficaces fondées sur l'utilisation de molécules inductrices de la différenciation pour obtenir, à partir de cet endoderme, une culture pure et massive d'hépatocytes ne présentant pas les inconvénients précédemment mentionnés. Les essais cliniques du BAL pourraient alors être relancés pour le traitement des insuffisances hépatiques aiguës avec plus de sécurité car il n'y a pas de contact direct entre les hépatocytes en culture dans le BRH et le patient, les échanges s'effectuant au travers d'une membrane semi-perméable. En outre, il serait inutile d'entreprendre une

${ }^{3}$ de la famille TGF- $\beta$. immunodépression permanente contrairement à la transplantation d'hépatocytes dans le foie d'un patient pour remplacer la population déficiente. Diverses pathologies hépatiques moins graves que l'hépatite fulminante (insuffisance hépatique chronique et maladies métaboliques du foie) pourraient aussi bénéficier de l'apport de cellules hépatiques différenciées à partir des hESC. $\diamond$

Human embryonic stem cells

to rescue fulminant hepatic failure

\section{RÉFÉRENCES}

1. Park JK, Lee DH. Bioartificial liver systems : current status and future perspective. J Biosci Bioeng 2005; 99: 311-9.

2. Sussman NL, Chong MG, Koussayer T, et al. Reversal of fulminant hepatic failure using an extracorporeal liver assist device. Hepatology $1992 ; 16: 60-5$.

3. Rozga J, Williams F, Ro MS, et al. Development of a bioartificial liver: properties and function of a hollowfiber module inoculated with liver cells. Hepatology $1993 ; 17: 258-65$.

4. Chamuleau RA, Deurholt T, Hoekstra R. Which are the right cells to be used in a bioartificial liver? Metab Brain Dis 2005; 20 : 327-35.

5. Paradis K, Langford G, Long Z, et al. Search for crossspecies transmission of porcine endogenous retrovirus in patients treated with living pig tissue. The Xen 111 study group. Science 1999 ; 285 : 1236-41.

6. Samuel D, Ichai P, Feray C, et al. Neurological improvement during bioartificial liver sessions in patients with acute liver failure awaiting transplantation. Transplantation $2002 ; 73: 257-64$.
7. Demetriou AA, Brown RS Jr, Busuttil RW, et al. Prospective, randomized, multicenter, controlled trial of a bioartificial liver in treating acute liver failure. Ann Surg 2004; 239: 660-70.

8. Stojkovic M, Lako M, Strachan T, Murdoch A. Derivation, growth and applications of human embryonic stem cells. Reproduction 2004 ; 128 : 259-67.

9. Thomson JA, Itskovitz-Eldor J, Shapiro SS, et al. Embryonic stem cell lines derived from human blastocysts. Science $1998 ; 282$ : 1145-7.

10. Lavon N, Benvenisty N. Study of hepatocyte differentiation using embryonic stem cells. J Cell Biochem 2005; 96 : 1193-202.

11. Lavon N, Yanuka O, Benvenisty N. Differentiation and isolation of hepatic-like cells from human embryonic stem cells. Differentiation $2004 ; 72: 230-8$.

12. Rambhatla L, Chiu CP, Kundu P, et al. Generation of hepatocyte-like cells from human embryonic stem cells. Cell Transplant 2003; 12 : 1-11.

13. Shirahashi H, Wu J, Yamamoto N, et al. Differentiation of human and mouse embryonic stem cells along a hepatocyte lineage. Cell Transplant 2004 ; 13: 197-211.

14. Schwartz RE, Linehan JL, Painschab MS, et al. Defined conditions for development of functional hepatic cells from human embryonic stem cells. Stem Cells Dev 2005; $14: 643-55$.

15. Yasunaga M, Tada S, Torikai-Nishikawa S, et al. Induction and monitoring of definitive and visceral endoderm differentiation of mouse ES cells. Nat Biotechnol 2005; 23 : 1542-50.

16. Kania G, Blyszczuk P, Jochheim A, et al. Generation of glycogen- and albumin-producing hepatocyte-like cells from embryonic stem cells. Biol Chem 2004; $385: 943-53$

17. Teratani T, Yamamoto $H$, Aoyagi $K$, et al. Direct hepatic fate specification from mouse embryonic stem cells. Hepatology $2005 ; 41: 836-46$.

18. D’Amour KA, Agulnick AD, Eliazer $S$, et al. Efficient differentiation of human embryonic stem cells to definitive endoderm. Nat Biotechnol 2005 ; 23 : 1534-41.

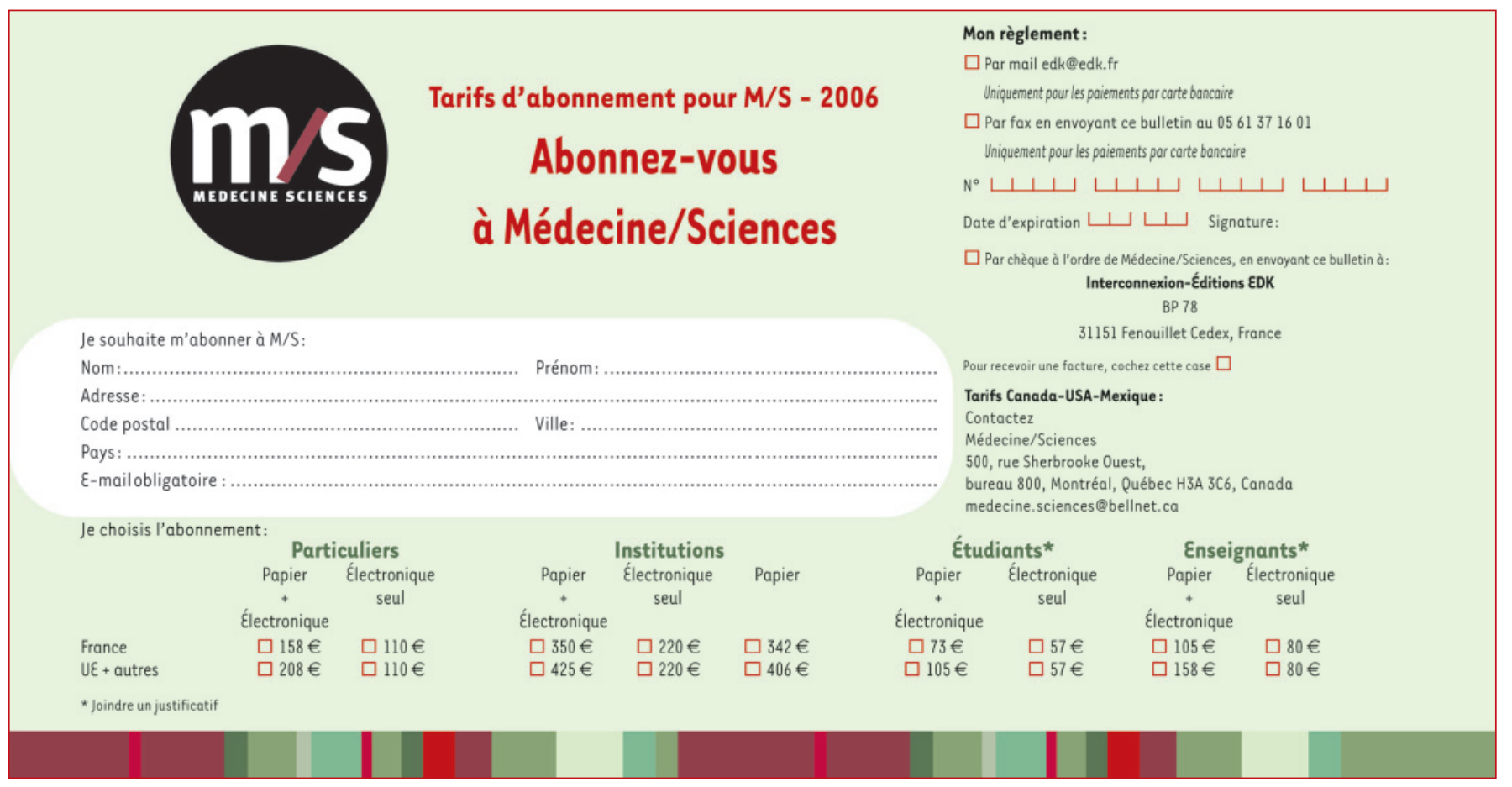

